\title{
Assessment tools for determining appropriateness of admission to acute care of persons transferred from long-term care facilities: a systematic review
}

\author{
Anna Renom-Guiteras ${ }^{1,2,3^{*}}$, Lisbeth Uhrenfeldt ${ }^{4}$, Gabriele Meyer ${ }^{1,5^{*}}$ and Eva Mann ${ }^{6}$
}

\begin{abstract}
Background: Residents of long-term care facilities have a high risk of acute care admission. Estimates of the frequency of inappropriate transfers vary substantially throughout the studies and various assessment tools have been used. The purpose of this study is to systematically review and describe the internationally existing assessment tools used for determining appropriateness of hospital admissions among long-term care residents.

Method: Systematic review of the literature of two databases (PubMed and CINAHL ${ }^{\oplus}$ ). The search covered seven languages and the period between January 2000 and December 2012. All quantitative studies were included if any assessment tool for appropriateness of hospital and/or emergency department admission of long-term care residents was used. Two pairs of independent researchers extracted the data.

Results: Twenty-nine articles were included, covering study periods between 1991 and 2009. The proportion of admissions considered as inappropriate ranged from 2\% to 77\%. Throughout the studies, 16 different assessment tools were used; all were based on expert opinion to some extent; six also took into account published literature or interpretation of patient data. Variation between tools depended on the concepts studied, format and application, and aspects evaluated. Overall, the assessment tools covered six aspects: specific medical diagnoses (assessed by $n=8$ tools), acuteness/severity of symptoms $(n=7)$, residents' characteristics prior to admission $(n=6)$, residents' or families' wishes $(n=3)$, existence of a care plan $(n=1)$, and availability or requirement of resources $(n=10)$. Most tools judged appropriateness based on one fulfilled item; five tools judged appropriateness based on a balance of aspects. Five tools covered only one of these aspects and only six considered four or more aspects. Little information was available on the psychometric properties of the tools.

Conclusions: Most assessment tools are not comprehensive and do not take into account residents' individual aspects, such as characteristics of residents prior to admission and wishes of residents or families. The generalizability of the existing tools is unknown. Further research is needed to develop a tool that is evidence-based, comprehensive and generalizable to different regions or countries in order to assess the appropriateness of hospital admissions among long-term care residents.
\end{abstract}

Keywords: Nursing home, Patient transfer, Hospitalization, Systematic review

\footnotetext{
* Correspondence: Anna.Renom@uni-wh.de; Gabriele.Meyer@medizin.uni-halle.de

${ }^{3}$ Institute of General Practice and Family Medicine, Faculty of Health,

University of Witten/Herdecke, Alfred-Herrhausen-Str. 50, Witten D-58448,

Germany

${ }^{5}$ Medical Faculty, Institute of Health and Nursing Science, Martin-Luther-University Halle-Wittenberg, Magdeburger Straße 8, Halle (Saale) D-06112, Germany

Full list of author information is available at the end of the article
}

\section{Ciomed Central}

( 2014 Renom-Guiteras et al.; licensee BioMed Central Ltd. This is an Open Access article distributed under the terms of the Creative Commons Attribution License (http://creativecommons.org/licenses/by/2.0), which permits unrestricted use, distribution, and reproduction in any medium, provided the original work is properly credited. The Creative Commons Public Domain Dedication waiver (http://creativecommons.org/publicdomain/zero/1.0/) applies to the data made available in this article, unless otherwise stated. 


\section{Background}

Residents of long-term care (LTC) facilities have a high risk of being admitted to hospital. Internationally, the incidence of visits to an emergency department has been estimated to be approximately 30 transfers per 100 LTC beds per year [1]. LTC residents are often sent to emergency departments (ED) when they are in a highly acute condition, and are likely to be admitted to the hospital [2]. Common underlying diagnoses are pneumonia, urinary tract infection, congestive heart failure, chronic obstructive pulmonary disease, fall-related injuries, and altered conscious state $[3,4]$.

LTC residents are often frail and suffer from diseases in advanced stages, have several comorbidities, high levels of dependency and take multiple medications. The referral or admission to an ED or acute hospital - although often unavoidable and beneficial - represents an unfavourable discontinuity of care and encompasses threats to the residents including distress, risk of iatrogenic events [5], and deterioration of mobility and cognition [6,7]. Beyond adverse clinical effects, hospital transfers account for a high proportion of total healthcare costs [8].

Many authors have evaluated the appropriateness of ED visits or hospitalisation among LTC residents. There is an on-going debate on how to define appropriateness of admissions in order to reduce negative effects of inappropriate transfers without withholding residents from admission if acute care is needed. To distinguish between admissions to acute care that are inappropriate and those that are not is of great interest not only for the residents concerned but also for nursing home providers and policy makers alike. In international studies, between $10 \%$ and $60 \%$ of hospital admissions have been classified as inappropriate $[9,10]$. So far, the reason for this high variability is not clarified. Variations may result from different study objectives, including different concepts such as inappropriate, preventable, avoidable, or unnecessary hospitalisation. Differences in acute care destinations and nursing home populations included in the studies may also affect the rates of inappropriate admissions. Several studies suggest that facility characteristics may be as important as residents' clinical characteristics [11,12]. In addition, regional differences in terms of financial incentives may also have an influence [13]. Interestingly, considerable variations in inappropriate hospital admission rates were even found in studies including nursing homes in well-defined areas only [14].

It is also important to take into account that authors used different assessment tools to judge the appropriateness of acute care transfers. Up to now, there is no consensus on which tool to use for assessment of appropriateness of residents' hospital admission. Furthermore, there is no agreement on the aspects to be covered by such a tool. The terminology and definitions are not yet clarified, as claimed by some authors [11,15-17]. As a first step towards clarification, it seems to be justified to systematically review all assessment instruments applied for judgement of appropriateness of transfers, to analyse their development, their underlying concepts, the aspects included, their psychometric properties, and to critically review them in the context of the complexity of acute care admissions of frail and vulnerable LTC residents.

Thus, the aim of our systematic review is 1) to provide an overview of the studies dealing with tools for assessing appropriateness of hospital admissions in LTC residents and 2) to describe the published assessment tools in detail, including information about their development and the aspects covered by the tools.

\section{Methods}

Four researchers from Spain, Germany, Denmark and Austria, all experienced in geriatric care and research, established a working group and developed a research protocol (available from the authors on request). In January 2013, two reviewers conducted a literature search. The search covered the databases Medline via PubMed and $\mathrm{CINAHL}^{\circ}$ and was limited to studies published between January 2000 and December 2012. The following search strategy was used for Pubmed: (("Residential Facilities"[MeSH]) OR (nursing homes) OR (homes for the aged) OR (aged care facilit*) OR (nursing facilit*) OR ("Long-Term Care"[MeSH])) AND (("Emergency Service, Hospital"[MeSH]) OR hospital OR (acute care) OR (emergency AND (medicine OR department* OR unit* OR ward $^{*}$ OR service* OR room*))) AND (appropriat* OR suitable OR avoidable OR preventable) AND (("Patient Transfer"[MeSH]) OR ("Hospitalization"[MeSH]) OR refer-

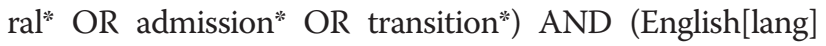
OR French[lang] OR German[lang] OR Spanish[lang] OR Catalan[lang] OR Danish[lang] OR Norwegian[lang]) AND ("2000/01/01"[PDat]: "2010"[PDat])). The corresponding search terms were used for CINAHL. Articles published in English, German, French, Spanish, Catalan, Danish and Norwegian were considered for inclusion. Two reviewers independently checked titles and abstracts for relevance and, in a second step, eligible full-text articles for inclusion. Reference lists of the included articles were checked manually. In addition, we followed PubMed-indexed related citations of two included articles which have been published recently and which focus on different acute care destinations $[10,15]$.

We included prospective and retrospective, experimental and non-experimental studies if they 1) investigated residents from any type of LTC setting who were transferred to hospital emergency departments or hospital wards, 2) provided or assessed diagnostic and/or therapeutic data on the process of transfer, 3) developed, administered or derived a tool for assessing appropriateness 
of hospital admissions, including any list of aspects or any single question that could be used to distinguish between appropriate or inappropriate admissions. Studies using different terms (e.g. inappropriate, preventable, avoidable admissions) and operational definitions of appropriateness were considered for inclusion.

Two pairs of independent researchers extracted information on the study characteristics and the assessment tools using a piloted data extraction form. Publications cited in the reference list were retrieved if necessary. Results were discussed and, in the case of disagreement, a third author was consulted to reach consensus. In case of doubt, the authors of the primary study were contacted.

Data extraction covered information about the type of study, description of participants and settings, information on which assessment tool was used, how and by whom it was used, number and proportion of inappropriate admissions to acute care reported, period of time studied, and information on how the assessment tool was developed and which items were evaluated by the tool. Once data extraction was finished, the research team agreed on a list of aspects that were covered by the items found in the assessment tools.

We refrained from formal critical appraisal of the included studies, since we were interested in the concepts and tools used for assessing appropriateness of hospital admissions only, rather than the internal validity of the studies. Assessment of risk of bias would not have provided any substantial information with regard to the aim of this review.

Inter-rater reliability was not calculated because most information extracted was descriptive. All disagreements could be solved after checking for accuracy and discussion.

\section{Results}

Twenty-nine articles met the inclusion criteria [3,4,8-10,15,18-41]. Two articles reporting on the same study were considered as one source [21,38]. A list of studies excluded, along with the reason for exclusion, is available from the authors on request. Figure 1 displays the process of identification of studies for inclusion in the systematic review. (Additional file 1: Table S1) presents the characteristics of the included studies. The majority $(n=24)$ were retrospective. Five studies reported on an intervention or a strategy for reducing transfers to acute care (information not shown in the table) $[21,23,26,27,30,38]$.

The majority of the studies $(n=24)$ investigated residents of LTC facilities only; five studies also included older persons living in the community [27,29,31,32,39]. Most studies $(n=25)$ considered the general population of LTC residents; four studies focused on specific groups: residents with long-term neurological conditions [32], residents with advanced cognitive impairment [37], and residents at the end-of-life [31,39]. Mean age of the study samples ranged from 81 [31,41] to 86 years [37], and the proportion of women varied from $62 \%$ [15] to $80 \%$ [23].

While types of LTC facilities seemed to be similar, the acute care destinations varied substantially: some studies focused either on ED visits or in-patient hospitalisation $(\mathrm{n}=3)$, others included in-patient hospitalisation only, irrespective of a previous ED visit $(n=8)$, others included ED visits with consecutive in-patient hospitalisation $(n=2)$, ED visits with subsequent discharge to nursing homes $(n=1)$ or ED visits irrespective of subsequent in-patient hospitalisation $(n=6)$. Some studies investigated hospitalisation without any further specification $(n=9)$.

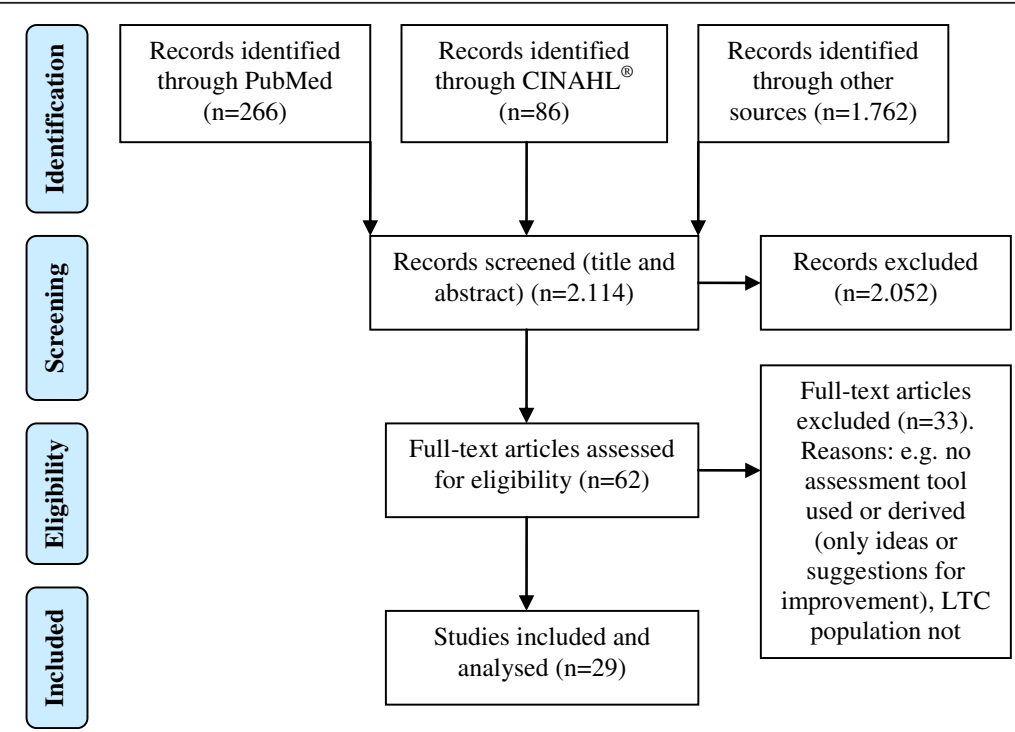

Figure 1 Identification of studies for inclusion in the systematic review. 
In eighteen studies the assessment tool used for determining appropriateness was applied to administrative databases. In eleven studies hospital or LTC facility records, or interviews with residents or nursing staff were used as data sources.

Results regarding the rate of inappropriate hospital admissions varied substantially. Some studies reported low proportions of inappropriate admissions. For example, Bermejo et al. [35] and Finn et al. [3] reported on 1.6\% and $13.1 \%$ of inappropriate emergency department visits, respectively; Becker et al. [33] reported on 18\% of preventable hospitalisation. Other studies documented high proportions of inappropriate admissions. In the study by Saliba et al. [18], 36\% of all ED visits were judged as inappropriate; Walker et al. [19] and Ouslander et al. [30] reported on $55 \%$ and $77 \%$ of potentially avoidable hospitalisation, respectively.

Sixteen assessment tools determining appropriateness of hospital admissions among residents of LTC facilities were identified throughout the included studies. Information on their names, development, psychometric properties, aim/concept studied, way of use, items included and aspects covered are displayed in (Additional file 2: Table S2). Those tools without an own name are given the name of the first author of the corresponding study (see column "Tool [corresponding studies]").

The terms used for indicating "inappropriate" hospitalisation varied throughout the different assessment tools: while most of them favoured the term "appropriate"/ "inappropriate" (e.g., AEP), others used the terms "avoidable" or "preventable" (e.g., ACSC; additional tool by Finucane et al. [9]; AHC), and one study applied the term "potentially burdensome" (tool by Gonzalo et al. [37]).

Most tools aimed at measuring appropriateness of hospital transfer, i.e., from the LTC facility to either ED or hospital ward. Some of them focused on visits to ED (e.g., Modified AEP, tool by Jensen et al. [15]), while others focused on admissions to hospital (e.g., AEP), or on both ED visits and hospital stay (e.g. Quality Improvement Review tool (INTERACT-II)). A smaller number of tools aimed at determining those hospital transfers which could have been prevented by adequate ambulatory care (e.g., ACSC, AHC), focusing therefore on the period preceding the acute moment of transition.

All assessment tools were developed and based upon expert opinion to different extents: two tools were compiled using an expert consensus method, and six expert groups also took into account the results of a literature search or the interpretation of patient data. In all studies, tools were applied retrospectively, i.e., after hospital admission had already taken place.

Assessment tools were applied by the investigators themselves $(n=9)$, an external panel of experts (generally with experience in LTC) looking for consensus $(n=5)$, or professionals directly engaged in the care of residents transferred $(\mathrm{n}=2)$.

As can be seen in Additional file 2: Table S2, some tools (e.g. AEP; ACSC) comprised a list of conditions or diseases (e.g. congestive heart failure, hypoglycaemia) while others consisted of a short definition or question (e.g. tool by Ong et al. [39], tool by Hammond et al. [32]).

The assessment tools differed widely regarding the aspects considered as criteria for judgement of appropriateness of acute care admissions. The six aspects are summarized in Table 1. Eight tools considered specific medical diagnoses as indicators for appropriate or inappropriate hospitalisation; seven tools considered the acuteness or severity of the symptoms at the moment of hospital transfer or admission; six tools took into account the resident's characteristics prior to admission; three tools considered the residents' or families' wishes; one tool assessed whether a nursing care plan had been defined and adhered to; ten tools considered resource availability or requirement.

While most tools judged appropriateness based on one fulfilled item of the above mentioned aspects, five tools determined appropriateness by considering a balance of issues, for example by asking the professionals applying the criteria to give their judgement on appropriateness after considering all the aspects.

Some tools focused on one or two of the aspects (e.g. ACSC; tool by Gonzalo et al. [37]), while others were more comprehensive, i.e. covered a higher number of aspects. Six tools covered four aspects or more (e.g. tool by Abel et al. [31]; tool by Jensen et al. [15]; Quality Improvement Review tool; SIR).

Most tools $(n=10)$ were developed or adapted in the context of the actual studies, providing no information about their use in other studies or generalizability. Other tools had been used previously, but with an aim other than assessing appropriateness of admission to hospital (e.g. AEP). Finally, some tools had been developed or used only in one country or context (e.g. ACSC, Quality Improvement Review tool (INTERACT-II)). Moderate to good levels of inter-rater reliability were found for six tools (SIR; AEP; tool by Abel et al. [31]; tool by Hammond et al. [32]; tool by Codde et al. [34].

\section{Discussion}

We reviewed 29 studies applying 16 assessment tools aimed at determining the appropriateness or preventability of ED or hospital admissions of LTC residents.

The rates of admissions considered as inappropriate differed substantially throughout the studies from $2 \%$ [9] to $77 \%$ [30]. The studies included in our review, most of them retrospective in nature and thus susceptible for bias, were distinctive in many aspects. They varied considerably in study designs and objectives. Outcomes were defined in 
Table 1 Aspects covered by the assessment tools

\begin{tabular}{|c|c|c|}
\hline Aspect & Examples of items included in the tools & $\begin{array}{l}\text { Number of tools } \\
\text { covering the aspect }\end{array}$ \\
\hline Specific medical diagnoses & $\begin{array}{l}\text { Suspected fracture, ACSC (asthma, congestive heart failure, angina, grand mal } \\
\text { seizure disorder, hypoglycaemia, hypertension, etc.), death }\end{array}$ & 8 \\
\hline $\begin{array}{l}\text { Acuteness or severity of symptoms } \\
\text { at time of transition }\end{array}$ & $\begin{array}{l}\text { Sudden onset of unconsciousness, incapacitating pain, tachycardia, gastrointestinal } \\
\text { bleeding symptoms, signs of being systemically unwell }\end{array}$ & 7 \\
\hline $\begin{array}{l}\text { Resident's characteristics prior } \\
\text { to admission to hospital }\end{array}$ & $\begin{array}{l}\text { Resident's baseline health status, level of functional ability, resident with advanced } \\
\text { cognitive impairment, presence of a terminal illness }\end{array}$ & 6 \\
\hline Resource availability/requirement & $\begin{array}{l}\text { Requirement of intravenous antibiotics, laboratory, radiology, admission to hospital, } \\
\text { physician and nurse availability and expertise }\end{array}$ & 10 \\
\hline Residents'/families' wishes & $\begin{array}{l}\text { Advance care directive in place, request of hospital admission or emergency } \\
\text { department visit by family }\end{array}$ & 3 \\
\hline $\begin{array}{l}\text { Information on the existence } \\
\text { of a care plan }\end{array}$ & Actions taken by staff before the transfer (including presence of advanced care planning) & 1 \\
\hline
\end{tabular}

different terms or even different concepts, e.g., inappropriate, avoidable, or preventable admissions. Besides, the acute care destinations varied, as well as the selection of the LTC population and LTC facility-level factors. Furthermore, studies took place in different regions and countries, implicating different reimbursement policies and financial incentives. The impact of these varying aspects on the rate of hospital admissions has been a matter of discussion for nearly 30 years. However, literature on this issue is scarce. In a previous review, case mix differences representing LTC population-level factors turned out to give only partial explanation for the variations in hospital admission [42]. This was confirmed by a study published by Wennberg et al., reporting that disparities in hospital admissions remained in similar geographic areas even after adjusting for case mix [43]. A recently published review of the literature confirmed that the propensity of being referred to acute care was rather associated with facility characteristics including nursing home ownership and bed-hold requirements than with patient characteristics [11].

Interestingly, to the best of our knowledge, the impact of assessment tools on the variability of inappropriate hospital admissions has not been studied so far.

In our review, we noticed considerable heterogeneity among the tools regarding the aims of use and the concepts studied (e.g. assessment of appropriateness of ED visits vs. in-patient hospitalisation; focus on preventable nature of the admissions vs. appropriateness of hospital transfer), format of use (tool applied by study authors vs. expert panel or nursing staff), data sources used (administrative databases vs. resident' hospital or LTC facility record vs. interview with residents or nursing staff), and aspects evaluated.

Our research team isolated six most prominent aspects considered by the assessment tools: specific medical diagnoses, acuteness or severity of symptoms at transition time point, resident's characteristics prior to admission to hospital, resource availability/requirement, residents'/families' wishes, information on the existence of a care plan. Most tools covered less than four aspects, and only six of them included four or more aspects and were therefore considered as more comprehensive. The individual aspects "residents' characteristics prior to admission to hospital" and "residents'/families' wishes" were evaluated only by six and three tools, respectively. Some tools (e.g. ACSC, Modified ACSC) only evaluated aspects like "specific medical diagnoses" or "acuteness or severity of symptoms at transition time point". Taking into consideration that residents in LTC facilities often differ in terms of comorbidity, cognitive and functional status, and stage of their diseases, it is surprising that residents' clinical characteristics prior to acute care admission were not acknowledged throughout as a necessary dimension of the judgement process. The same applies to residents' and relatives' preferences which otherwise play an important role regarding the present advocacy towards person-centred care [44]. It may also be seen as a weakness of the existing tools that they did not consistently include facility-level characteristics as an indicator of the appropriateness of admissions. In respect to the frequently quickly changing conditions of residents, the presence of skilled nursing staff and the availability of technical equipment including diagnostic and therapeutic procedures may greatly influence the decision on the appropriateness of acute care admission. Finally, only 5 tools judged appropriateness based on a balance of aspects.

All tools identified in this systematic review were developed based on expert opinion, at least to a great extent. Information on generalizability in other regions or countries is scarce.

Our findings are supported by a non-systematic review $[17,45]$. Ouslander and Maslow did not focus on LTC residents only, but also included community-dwelling older persons. The review on preventable hospitalisations focusses on 
U.S. information sources and perspectives. The authors emphasize, as we do, the need for comprehensive measures to account for aspects such as medical comorbidities, clinical complexity or differences in resources in the care settings. They also criticize the lack of attention to how and where decisions about hospitalisation are made.

Our systematic review focussed on the assessment of appropriateness among LTC residents. The assessment of appropriateness of hospital admission among communitydwelling older persons may require the consideration of similar aspects, but adapted to the different setting. To the best of our knowledge, no systematic review covering international studies on this issue is available so far.

It may be seen as a limitation that we did not systematically assess the risk of bias of the original studies included in our systematic review. However, we were interested in the concepts and tools used for assessing appropriateness of hospital admissions, rather than in the internal validity of the studies. Nevertheless, even without formal validity assessment, it is obvious that the included studies suffer from methodological shortcomings, since many used secondary or retrospective routine data analysis and are therefore more prone to bias.

Our review, which is the first to overview the tools internationally used to assess the appropriateness of hospital admissions among LTC residents, may contribute to the clarification of the concept "appropriateness of admission of LTC residents to acute care". It also may present a first step towards the development of an evidence-based, comprehensive and generalizable tool. Such a tool may have a two-fold function: first as a quality indicator to assess the appropriateness of the decisions made when admitting individual residents to acute care, considering that the resources available were not modifiable at that time, and secondly to identify areas of improvement such as the need for training in palliative care or the need for more resources. The tool may attempt to assess appropriateness minimizing the effects of the different rater perspectives (i.e. nursing staff of the LTC facility, ED professionals, and researchers). It may also be used to assess the effectiveness of new interventions aimed at improving appropriateness of transition of LTC residents to acute care.

In the meanwhile, studies aiming at assessing appropriateness of admitting LTC residents to hospital are encouraged to use an assessment tool according to predefined aims and taking the different aspects into consideration. Studies should mention why a certain tool was chosen and the limitations of not using a more comprehensive tool should be clearly mentioned.

\section{Conclusions}

Our systematic review analysed 29 studies assessing the prevalence of the appropriateness of acute care admissions, which varied widely throughout the studies. We found 16 different assessment tools used in the studies. Only six tools covered more than four aspects as criteria to determine the appropriateness of acute care admissions. Most assessment tools did not take into account residents' individual aspects, such as characteristics of residents prior to admission and wishes of residents or families. Tools were based mostly on expert opinion, and information on their generalizability is not provided. Further research is warranted to develop an evidence-based and comprehensive tool supported by quality assuring strategies to improve decisions on the appropriateness of ED and hospital admissions among residents of LTC facilities.

\section{Additional files}

Additional file 1: Table S1. Studies dealing with assessment tools for determining appropriateness of hospital admissions among residents of LTC facilities.

Additional file 2: Table S2. Characteristics of the assessment tools to determine appropriateness of hospital admissions among residents of LTC facilities [46-52].

\section{Competing interests}

The authors declare that they have no competing interests.

\section{Authors' contributions}

Review protocol: ARG, EM \& GM. Literature search: ARG, EM, LU \& GM. Data extraction: ARG, EM, LU \& GM. Data interpretation: ARG, GM, EM, LU. Drafting of the manuscript: ARG. Critical revision of the manuscript with regard to important intellectual content: GM, EM \& LU. Study supervision: EM \& GM. All authors read and approved the final manuscript.

\section{Acknowledgements}

We thank Mr Adriel Ortega, an exchange student from the USA at the University of Witten/Herdecke, who contributed to the literature search. We also thank Ms Karin Velbek, hospital library service at Horsens Hospital, who supported the literature search, and Ms Vivienne Krause, University of Witten/ Herdecke, who checked the manuscript for English language. We thank the Sociedad Española de Geriatría y Gerontología (Spanish Society of Geriatric Medicine and Gerontology) for the grant which supported the work of the first author of this study.

\section{Author details}

${ }^{1}$ School of Nursing Science, Faculty of Health, University of Witten/Herdecke, Witten, Germany. ${ }^{2}$ Universitat Autònoma de Barcelona, Barcelona, Spain. ${ }^{3}$ Institute of General Practice and Family Medicine, Faculty of Health, University of Witten/Herdecke, Alfred-Herrhausen-Str. 50, Witten D-58448, Germany. ${ }^{4}$ Department of Research, Horsens Hospital, Department of Public Health, Aarhus University, Aarhus, Denmark. ${ }^{5}$ Medical Faculty, Institute of Health and Nursing Science, Martin-Luther-University Halle-Wittenberg, Magdeburger Straße 8, Halle (Saale) D-06112, Germany. Institute of General Practice, Family Medicine and Preventive Medicine, Paracelsus Medical University, Salzburg, Austria.

Received: 31 January 2014 Accepted: 11 June 2014

Published: 22 June 2014

\section{References}

1. Arendts G, Howard K: The interface between residential aged care and the emergency department: a systematic review. Age Ageing 2010, 39:306-312.

2. Wang HE, Shah MN, Allman RM, Kilgore M: Emergency department visits by nursing home residents in the United States. J Am Geriatr Soc 2011, 59:1864-1872 
3. Finn JC, Flicker L, Mackenzie $E$, Jacobs IG, Fatovich DM, Drummond S, Harris M, Holman DC, Sprivulis P: Interface between residential aged care facilities and a teaching hospital emergency department in Western Australia. Med J Aust 2006, 184(9):432-435.

4. Gruneir A, Bell CM, Bronskill SE, Schull M, Anderson GM, Rochon PA: Frequency and pattern of emergency department visits by long-term care residents: a population-based study. J Am Geriatr Soc 2010, 58(3):510-517.

5. Steel K, Gertman PM, Crescenzi C, Anderson J: latrogenic illness on a general medical service at a university hospital. Qual Saf Health Care 2004, 13(1):76-80.

6. Foreman MD, Theis $\mathrm{SL}$, Anderson MA: Adverse events in the hospitalized elderly. Clin Nurs Res 1993, 2(3):360-370.

7. Wakefield BJ: Risk for acute confusion on hospital admission. Clin Nurs Res 2002, 11(2):153-172

8. Grabowski DC, O'Malley AJ, Barhydt NR: The costs and potential savings associated with nursing home hospitalizations. Health Aff (Millwood) 2007, 26(6):1753-1761.

9. Finucane $P$, Wundke R, Whitehead C, Williamson L, Baggoley C: Use of in-patient hospital beds by people living in residential care. Gerontology 2000, 46(3):133-138.

10. Ouslander JG, Lamb G, Perloe M, Givens JH, Kluge L, Rutland T, Atherly A, Saliba D: Potentially avoidable hospitalizations of nursing home residents: frequency, cause, and costs. J Am Geriatr Soc 2010, 58(4):627-635.

11. Grabowski DC, Stewart KA, Broderick SM, Coots LA: Predictors of nursing home hospitalization: a review of the literature. Med Care Res Rev 2008, 65(1):3-39.

12. Carter MW, Porell FW: Variations in hospitalization rates among nursing home residents: the role of facility and market attributes. The Gerontologist 2003, 43(2):175-191.

13. Grabowski DC: Medicate and Medicaid: conflicting incentives for long-term care. Milbank Q 2007, 85:579-610.

14. Graverholt B, Riise T, Jamtvedt G, Husebo BS, Nordtvedt MW: Acute hospita admissions from nursing homes: predictors of unwarranted variation? Scand J Public Health 2013, 41(4):359-365.

15. Jensen PM: Are long-term care residents referred appropriately to hospital emergency department? Can Fam Physician 2009, 55(5):500-505.

16. Jablonski RA, Utz SW, Steeves R, Gray DP: Decisions about transfer from nursing home to emergency department. J Nurs Scholarsh 2007, 39(3):266-272

17. Ouslander JG, Maslow K: Geriatrics and the triple aim: defining preventable hospitalizations in the long-term care population. J Am Geriatr Soc 2012, 60:2313-2318.

18. Saliba D, Kington R, Buchanan J, Bell R, Wang M, Lee M, Herbst M, Lee D Sur D, Rubenstein $L$ : Appropriateness of the decision to transfer nursing home facility residents to the hospital. J Am Geriatr Soc 2000, 48(2):154-163.

19. Walker JD, Teare GF, Hogan DB, Lewis S, Maxwell CJ: Identifying potentially avoidable hospital admissions from Canadian long-term care facilies. Med Care 2009, 47(2):250-254

20. Caffrey C: Potentially preventable emergency department visits by nursing home residents: United States, 2004. NCHS Data Brief 2010, 33:1-8.

21. Lamb G, Tappen R, Diaz S, Herndon L, Ouslander JG: Avoidability of hospital transfers of nursing home residents: perspectives of frontline staff. J Am Geriatr Soc 2011, 59(9):1665-1672.

22. Murtaugh CM, Litke A: Transitions through post-acute and long-term care settings: patterns of use and outcomes for a national cohort of elders. Med Care 2002, 40(3):227-236.

23. Kane RL, Keckhafer G, Flood S, Bershadsky B, Sladaty MS: The effect of evercare on hospital use. J Am Geriatr Soc 2003, 51(10):1427-1434.

24. Carter MW: Factors associated with ambulatory care-sensitive hospitalizations among nursing home residents. J Aging Health 2003, 15(2):295-331.

25. Intrator O, Zinn J, Mor V: Nursing home characteristics and potentially preventable hospitalizations of long-stay residents. J Am Geriatr Soc 2004 52(10):1730-1736.

26. Kane RL, Flood S, Bershadsky B, Keckhafer G: Effect of an innovative medicare managed care program on the quality of care for nursing home residents. Gerontologist 2004, 44(1):95-103.

27. Kane RL, Homyak P, Bershadsky B, Flood S, Zhang S: The quality of care under a managed-care program for dual eligible. Gerontologist 2005, 45(4):496-504.
28. Carter MW, Porell FW: Vulnerable populations at risk of potentially avoidable hospitalizations: the case of nursing home residents with Alzheimer's disease. Am J Alzheimers Dis Other Demen 2005, 20(6):349-358.

29. Carter MW, Datti B, Winters JM: ED visits by older adults for ambulatory care-sensitive and supply-sensitive conditions. Am J Emerg Med 2006, 24(4):428-434

30. Ouslander JG, Perloe M, Givens JH, Kluge L, Rutland T, Lamb G: Reducing potentially avoidable hospitalizations of nursing home residents: results of a pilot quality improvement project. J Am Med Dir Assoc 2009, 10(9):644-652.

31. Abel J, Rich A, Griffin T, Purdy S: End-of life care in hospital: a descriptive study of all inpatient deaths in 1 year. Palliat Med 2009, 23(7):616-622

32. Hammond $C L$, Phillips MF, Pinnington $L L$, Pearson $B J$, Fakis $A$ : Appropriateness of acute admissions and last in-patient day for patients with long-term neurological conditions. BMC Health Serv Res 2009, 9:40.

33. Becker MA, Boaz TL, Andel R, Gum AM, Papadopoulos AS: Predictors of preventable nursing home hospitalizations: the role of mental disorders and dementia. Am J Geriatr Psychiatry 2010, 18(6):475-482.

34. Codde J, Frankel J, Arendts G, Babich P: Quantification of the proportion of transfers from residential aged care facilities to the emergency department that could be avoided through improved primary care services. Australas J Ageing 2010, 29(4):167-171.

35. Bermejo Higuera JC, Carabias Maza R, Díaz-Albo Hermida E, Muñoz Alustiza C, Villacieros Durbán M: Derivaciones al Servicio de Urgencias del hospital en una población de ancianos residentes. Estudio retrospectivo sobre sus causas y adecuación. [Hospital transfers from a population of elderly residents. A retrospective study about the causes and suitability]. Gerokomos 2010, 21(3):114-117

36. Kada O, Brunner E, Likar R, Pinter G, Leutgeb I, Francisci N, Pfeiffer B, Janig $H$ : Vom Pflegeheim ins Krankenhaus und wieder zurück... eine multimethodale analyse von krankenhaustransporten aus alten- und pflegeheimen. [from the nursing home to hospital and back again... a mixed methods study on hospital transfers from nursing homes]. Z Evid Fortbild Qual Gesundhwes 2011, 105(10):714-722. doi: 10.1016/j.zefq.2011.03.023. Epub 2011 Apr 13

37. Gonzalo P, Teno JM, Mitchell SL, Skinner J, Bynum J, Tyler D, Mor V: End-oflife transitions among nursing home residents with cognitive issues. N Engl J Med 2011, 365:1212-1221.

38. Ouslander JG, Lamb G, Tappen R, Herndon L, Diaz S, Roos BA, Grabowsk $D C$, Bonner $A$ : Interventions to reduce hospitalizations from nursing homes: evaluation of the interact ii collaborative quality improvement project. J Am Geriatr Soc 2011, 59(4):745-753.

39. Ong AC, Sabanathan K, Potter JF, Myint PK: High mortality of older patients admitted to hospital from care homes and insight into potential interventions to reduce hospital admissions from care homes: the Norfolk experience. Arch Gerontol Geriatr 2011, 53(3):316-319.

40. Young Y, Inamdar S, Dichter BS, Kilburn HJ, Hannan EL: Clinical and nonclinical factors associated with potentially preventable hospitalizations among residents in New York State. J Am Med Dir Assoc 2011, 12(5):364-371.

41. Becker M, Boaz T, Andel R, DeMuth A: Predictors of avoidable hospitalizations among assisted living residents. J Am Med Dir Assoc 2012, 13(4):355-359. Doi: 10.1016/j.jamda.2011.02.001. Epub 2011 Mar 12.

42. Castle NG, Mor V: Hospitalization of nursing home residents: a review of the literature, 1980-1995. Med Care Res 1996, 53(2):123-148.

43. Wennberg J: Unwarranted variations in healthcare delivery: implications for academic medical centres. BMJ 2002, 325(7370):961-964.

44. Olsson LE, Jakobsson Ung E, Swedberg K, Ekman I: Efficacy of personcentred care as an intervention in controlled trials - a systematic review. J Clin Nurs 2013, 22(3-4):456-465. doi:10.1111/jocn.12039. Epub 2012 Dec 12

45. Maslow K, Ouslander JG: Measurement of potentially preventable hospitalizations, long-term quality alliance, 2012. [http://www.ltqa.org/wpcontent/themes/ItqaMain/custom/images//PreventableHospitalizations_ 021512_2.pdf] Accessed on January 30, 2014

46. Gertman PM, Restuccia JD: The appropriateness evaluation protocol: a technique for assessing unnecessary days of hospital care. Med Care 1981, 19(8):855-871.

47. Baggoley CJ, Phillips DG, Aplin PJ: A study of emergency admissions at the flinders medical centre using the appropriateness evaluation protocol. Emerg Med Australas 1994, 6:1-89. 
48. Weissman J, Constantine G, Epstein A: Rates of avoidable hospitalization by insurance status in Massachusetts and Maryland. JAMA 1992, 268:2388-2394.

49. Billings J, Zeitel L, Lukomnik J, Carey TS, Blank AE, Newman L: Impact of socioeconomic status on hospital use in New York City. Health Aff (Millwood) 1993, 12(1):162-173.

50. Blustein J, Hanson K, Shea S: Preventable hospitalizations and socioeconomic status. Health Aff (Millwood) 1998, 17(2):177-189.

51. Department of Health: End of Life Care Strategy - promoting high quality care for all adults at the end of life. 2008, [https:/www.gov.uk/

government/uploads/system/uploads/attachment_data/file/136431/ End_of_life_strategy.pdf] Accessed on July 29, 2013.

52. Wetle T, Teno J, Shield R, Welch L, Miller S: End Of Life In Nursing Homes: Experiences And Policy Recommendations. Washington, DC: AARP; 2004. [http://assets.aarp.org/rgcenter/health/2004_14_eol.pdf] Accessed on May 30, 2013.

doi:10.1186/1471-2318-14-80

Cite this article as: Renom-Guiteras et al:: Assessment tools for determining appropriateness of admission to acute care of persons transferred from long-term care facilities: a systematic review. BMC Geriatrics 2014 14:80.

\section{Submit your next manuscript to BioMed Central and take full advantage of:}

- Convenient online submission

- Thorough peer review

- No space constraints or color figure charges

- Immediate publication on acceptance

- Inclusion in PubMed, CAS, Scopus and Google Scholar

- Research which is freely available for redistribution 